\title{
A utilização do múltiplo EV/EBITDA na precificação de IPO's no mercado brasileiro
}

\author{
Valuing IPO's using EV/EBITDA multiple in the Brazilian market
}

La utilización del múltiple EV/EBITDA en la valorización de las IPO's en el mercado brasileño

\section{Anatoli Dias Orlovas}

Mestre em Administração na Fundação Escola de Comércio Álvares Penteado (FECAP) Endereço: Av. Goias, ${ }^{\circ}$ 1805, Bairro Santa Paula CEP: 09521-300 - São Caetano do Sul/SP - Brasil E-mail: anatoli.orlovas@gmail.com

Telefone: (11) 4239-6128

\section{Ricardo Goulart Serra}

Pós-doutor em Ciências Contábeis na Universidade São Paulo (FEA/USP)

Professor do MPA da Fundação Escola de Comércio Álvares Penteado (FECAP), Professor da Pós-graduação Lato Sensu do Instituto de Ensino e Pesquisa (Insper) e Professor da Graduação do Departamento de Administração da Universidade de São Paulo (FEA/USP) Endereço: Avenida da Liberdade, 532, Liberdade

CEP: 01502-001 - São Paulo/SP - Brasil

E-mail: ricardo.serra@usp.br

Telefone: (11) 3272-2301

\section{Liliam Sanchez Carrete}

Doutor em Administração na Universidade de São Paulo (USP)

Professor do Departamento de Administração da Universidade de São Paulo (FEA/USP)

Endereço: Avenida Professor Luciano Gualberto, 908, FEA 1, sala G-168, Cidade

Universitária

CEP: 05508-900 - São Paulo/SP - Brasil

E-mail: 1scarrete@usp.br

Telefone: (11) 3091-5956

Artigo recebido em 25/09/2017. Revisado por pares em 16/04/2018. Reformulado em 01/07/2018. Recomendado para publicação em 14/11/2018 por Carlos Eduardo Facin Lavarda Editor-Chefe). Publicado em 10/04/2019. 


\section{Resumo}

Este artigo verificou se o múltiplo EV/EBITDA de empresas comparáveis (pertencentes ao mesmo setor) são bons parâmetros para precificar os IPO's (initial public offering). Foram 116 IPO's no mercado brasileiro, entre 2004 e 2014. Após as exclusões (empresas financeiras, empresas com falta de informações ou informações negativas e outliers), foram analisados 36 IPO's. Além dos múltiplos das empresas comparáveis, utilizou-se como variáveis explicativas: dummy de ano e variáveis ligadas aos drivers de valor (rentabilidade, alavancagem e tamanho). Os resultados indicam que o múltiplo de empresas comparáveis é relevante para precificar as empresas no momento do IPO, sendo que os modelos com a média e com a mediana obtiveram melhores coeficiente de ajuste $\left(\mathrm{R}^{2}\right)$. Nenhum dos drivers de valor mostrou-se significativo. A dummy de ano foi significativa apenas para um modelo. Os modelos com a classificação setorial NAICS apresentaram ajustes melhores do que os modelos com a classificação da Economatica ${ }^{\circledR}$.

Palavras-chave: IPO; EV/EBITDA; Avaliação por múltiplos; Avaliação de empresas

\section{Abstract}

This paper verified whether the EV / EBITDA multiple of comparable firms (belonging to the same industry) are good parameters for pricing firms in their initial public offering (IPO's). There were 116 IPO's in the Brazilian market between 2004 and 2014. After the exclusions (financial firms, firms with lack of or negative information and outliers), 36 IPO's were analyzed. In addition to the multiples of the comparable firms, it was used as explanatory variables: year dummy and variables related to value drivers (return, leverage and size). The results indicate that the multiple of comparable firms are relevant to price the firms 'going public', and the models with the mean and the median obtained better adjustment coefficient $\left(R^{2}\right)$. The analyzed value drivers were not significant. The year dummy was significant only for one model. The models with the NAICS' sector classification presented better adjustments than the models with the Economatica's ${ }^{\circledR}$ classification.

Keywords: IPO; EV/EBITDA; Multiple's valuation; Valuation

\section{Resumen}

Este artículo verificó si el múltiplo EV / EBITDA de empresas comparables (pertenecientes al mismo sector) son buenos parámetros para valorizar las IPO (initial public offering) en el mercado brasileño, entre 2004 y 2014. Después de las exclusiones (empresas financieras, empresas con falta de información o información negativa y outliers), se analizaron 36 IPO's. Además de los múltiples de las empresas comparables, se utilizó como variables explicativas: dummy de año y variables ligadas a los controladores de valor (rentabilidad, apalancamiento y tamaño). Los resultados indican que el múltiplo de empresas comparables es relevante para valorizar a las empresas en el momento de la IPO, siendo que los modelos con la media y con la mediana obtuvieron mejores coeficientes de ajuste (R2). Ninguno de los controladores de valor se mostró significativo. La simulación de cada año fue significativa sólo para un modelo. Los modelos con la clasificación sectorial NAICS presentaron ajustes mejores que los modelos con la clasificación de Economatica ${ }^{\circledR}$.

Palabras clave: IPO; EV/EBITDA; Evaluación por múltiples; Evaluación de empresas

\section{Introdução}

Entre 2004 a 2014 houve 116 IPO's (Initial Public Offering) no mercado acionário brasileiro. Fatores como a estabilização político-econômica, o aumento do investimento de capital estrangeiro, a criação do Novo Mercado, a profissionalização da Bovespa, entre outros 
(CASOTTI; MOTTA, 2008), contribuíram para o elevado número de IPO's neste período, embora a recente piora do cenário macroeconômico tenha feito com que o número de IPO's diminuísse, chegando a zero em 2015.

IPO é a denominação dada ao momento em que uma empresa realiza, pela primeira vez, uma oferta pública de ações ao mercado - Oferta Pública Inicial, transformando-a em uma empresa listada na bolsa de valores. Diversos motivos fazem com que uma empresa realize a primeira oferta pública, entre eles: captar recursos, obter maior reconhecimento do mercado, profissionalizar sua gestão, viabilizar a troca de sócios e investidores, possibilitar a utilização de suas próprias ações para adquirir outras empresas etc.

O momento da entrada de uma empresa na bolsa de valores gera expectativas tanto por parte da empresa, que espera obter os recursos estimados vendendo suas ações, bem como por parte dos investidores, que esperam obter retornos com a decisão de investir nesta mesma empresa (RITTER, 1991). Sendo assim, a avaliação de empresas no momento do seu IPO é uma tarefa crítica, a qual será avaliada neste trabalho.

Pode-se avaliar empresas por diversas técnicas (avaliação por fluxo de caixa descontado, avaliação por múltiplo etc.) que normalmente são utilizados em conjunto. Embora dificultada pela menor disponibilidade de informações das empresas fechadas comparativamente às empresas abertas, a técnica de avaliação por fluxo de caixa descontado é aplicável para avaliá-las. A técnica de avaliação de empresas por múltiplos de empresas comparáveis, por sua vez, é amplamente recomendada (KIM; RITTER, 1999), sendo este o método mais utilizado em estudos de avaliação de empresas durante o momento da oferta inicial (CASOTTI; MOTTA, 2008). Este método utiliza múltiplos de empresas parecidas (comparáveis ou peers) como base para a precificação da empresa que está sendo avaliada. Um múltiplo pode ser descrito como a razão entre o preço (enterprise value ou equity value, conforme o caso) dividido por um parâmetro da empresa (geralmente contábil, como EBITDA - lucro antes dos impostos, juros, depreciação e amortização, lucro líquido ou valor patrimonial).

A questão de pesquisa que motiva o presente artigo é se os múltiplos das empresas comparáveis explicam os múltiplos implícitos na precificação dos IPO's no mercado brasileiro. Portanto, o objetivo do trabalho é verificar se, no momento do IPO, os múltiplos das empresas comparáveis (pertencentes ao mesmo setor da empresa participante do IPO) são bons parâmetros para precificar um IPO. Sendo assim, o trabalho testou a seguinte hipótese:

Hipótese: os múltiplos das empresas comparáveis (múltiplos das peers) explicam o múltiplo implícito na precificação dos IPO's (múltiplos IPO's) de empresas brasileiras.

A hipótese foi testada em dois momentos distintos: (i) o dia do IPO (D0, base para o preço inicial do IPO, o qual é referenciado no prospecto final do IPO) e (ii) o fechamento do primeiro dia de negociação (D1). O preço no dia D0 é fruto do bookbuilding e o preço no dia D1 considera a efetiva negociação pública das ações.

Busca-se evidências empíricas da utilização da avaliação por múltiplos como alternativa para a avaliação de empresas "abrindo o capital".

Considerando o IPO da Natura, em 2004, como o ponto de inflexão a partir do qual os IPO's ressurgiram no Brasil, analisou-se o período iniciado em 2004. O fim do período é 2015, porém, como em 2015 não houve IPO's no Brasil, está-se considerando o fim do período em 2014 (onze anos). Ao todo, foram analisadas 36 IPO's. Estudou-se o múltiplo EV/EBITDA (Enterprise Value-to-EBITDA). Este múltiplo está entre os dois mais utilizados pelos praticantes de avaliação de empresas (FERNANDEZ, 2015), tendo sido, segundo Kim e Ritter (1999), o mais preciso na precificação de IPO's, entre as variáveis estudadas pelos autores. Baker e Rubak (1999) e Couto Jr. e Galdi (2012) encontram o mesmo resultado em situações gerais (não específicas a IPO). 
Este artigo contém esta seção introdutória, seguida da seção com a fundamentação teórica e da seção metodológica. As duas últimas seções apresentam a análise dos resultados e elaboram as considerações finais.

\section{Fundamentação Teórica}

Existem algumas técnicas utilizadas para avaliar uma empresa, por exemplo: avaliação por fluxo de caixa descontado, avaliação por múltiplos e avaliação por opções reais. A avaliação por múltiplos visa precificar uma empresa com base no preço de outra(s) empresa(s). Para tanto, define-se qual(is) empresa(s) é(são) parecida(s) com a empresa sendo avaliada, chamada(s) empresa(s) comparável(is) ou peer(s) e, por meio de uma triangulação de preço, precifica-se a empresa em questão (SERRA; WICKERT, 2014).

Para sua aplicação, (i) escolhe-se um parâmetro de uma empresa que tenha relação com o valor da mesma, por exemplo, o lucro líquido, (ii) identifica-se empresa(s) parecida(s) com a empresa sendo avaliada (peer $(s)$ ), (iii) forma-se, com o parâmetro identificado e o preço da(s) $\operatorname{peer}(s)$ o índice chamado de múltiplo (por exemplo, Preço/Lucro ou Price/Earnings), (iv) calcula-se a média (ou outra medida de posição, tais como: mediana e média harmônica) do(s) múltiplo(s) da(s) peer(s) e (v) aplica-se o múltiplo médio da(s) peer $(s)$ ao parâmetro da empresa sendo avaliada para obter o seu preço. Esta técnica é recomendável para avaliar ativos (LIE; LIE, 2002) e é extensamente utilizada por investidores e analistas de mercado (BHOJRAJ et al., 2002; DAMODARAN, 2006).

Os principais múltiplos utilizados são (FERNANDEZ, 2015): (i) Price/Earnings (P/E), obtido por meio da divisão do valor de mercado do patrimônio líquido (equity value) pelo lucro líquido (earnings) e (ii) $E V / E B I T D A$, obtido por meio da divisão do valor de mercado da empresa - equity value mais dívida líquida (enterprise value) pelo lucro antes de juros, impostos, depreciação e amortização (EBITDA) - o foco deste estudo. estudam:

No presente artigo, os trabalhos envolvendo múltiplos estão agrupados naqueles que

(i) Múltiplos como preditores de retorno,

(ii) Performance dos múltiplos na explicação do preço e

(iii) Identificação das empresas comparáveis (peers).

\subsection{Múltiplos como preditores de retorno}

Desde a formulação do CAPM por Sharpe (1964), Lintner (1965) e Black (1972) que os acadêmicos testam (i) se o beta mede risco e (ii) se o beta mede todo o risco. Neste sentido, os estudos testam se outras variáveis adicionadas ao beta são capazes de explicar melhor o retorno das empresas. Entre as variáveis utilizadas (sozinhas ou em conjunto com outras variáveis) estão alguns múltiplos, sendo eles: P/E (BALL, 1978; REIGANUM, 1981; BOATSMAN; BASKIN, 1981; FAIRFIELD, 1994; PENMAN, 1996 entre outros), P/B (STATMAN, 1980; WILCOX, 1984; CHAN; HAMAO; LAKONISHOK, 1991; FAMA; FRENCH, 1992; FAIRFIELD, 1994; PENMAN, 1996 entre outros) e EV/EBITDA (LOUGHRAN; WELLMAN, 2010; WALKSHÄUSL; LOBE, 2015 entre outros). Diversos estudos encontraram significância na relação entre retorno e as variáveis estudadas. Por outro lado, estudiosos como Black (1993) contestam a validade de tais variáveis na explicação do retorno das ações.

$\mathrm{Na}$ tentativa de identificar se tal relação trata-se de uma anomalia (má precificação), alguns acadêmicos verificaram se o mercado aprende com os estudos acadêmicos: Jegadeesh e Titman (2001) documentam que os retornos anormais aumentaram após a divulgação do efeito momento e Schwet (2003), Malkiel (2014) e McLean e Pontiff (2016) verificaram que os 
retornos anormais caíram após a publicação de diversos estudos (os últimos autores tendo investigado o efeito após a publicação de 79 artigos). Uma possível explicação para a queda do retorno anormal é a de que a relação entre retorno e a variável estudada é derivada de uma anomalia.

\subsection{Performance dos múltiplos na explicação do preço}

Uma outra linha de pesquisa tem como objetivo identificar quais múltiplos têm a melhor performance na explicação do preço. Além dos diversos múltiplos pesquisados, os estudos também se diferenciam em utilizar (i) informações históricas ou (ii) informações projetadas.

As conclusões não são unânimes, tendo Lie e Lie (2002) indicado a preferência por múltiplos de ativo $(\mathrm{P} / \mathrm{B})$ em detrimento a múltiplos de venda ou lucro e a preferência por múltiplos de EBITDA em detrimento a múltiplos de EBIT (lucro antes de juros e impostos). Kim e Ritter (1999) e Baker e Ruback (1999) documentam a melhor performance dos múltiplos de EBITDA (os primeiros estudando IPO's). Liu, Nissim e Thomas (2002) hierarquizaram o múltiplo P/E como o que mostrou melhores resultados e Firth, Li e Wang (2008) documentaram boa performance do P/E ao analisarem IPO's na China.

Kaplan e Ruback (1995) defendem que não existe um método claro para identificar qual a melhor métrica a ser utilizada. Outros autores defendem que o setor de atuação da empresa pode influenciar no múltiplo que apresente melhor desempenho (DAMODARAN, 2006; FERNANDEZ, 2015). Há quem defenda a utilização conjunta de mais de um múltiplo (BHOJRAJ; LEE, 2002; BHOJRAJ; LEE; NG, 2003), como por exemplo P/E e P/B (CHENG; MCNAMARA, 2000).

No que diz respeito a utilização de informações históricas ou projetadas, Zarowin (1990), Alford (1992) e Liu, Nissim e Thomas (2002) indicam a melhor performance dos múltiplos de informações projetadas.

Couto Jr. e Galdi (2012) concluíram, analisando empresas brasileiras no período de 1994 a 2007, que o múltiplo P/EBITDA (Price/EBITDA) explica melhor o preço das ações comparativamente ao $\mathrm{P} / \mathrm{E}, \mathrm{P} / \mathrm{B}$ e $\mathrm{P} / \mathrm{S}$ (Price/Sale ou preço dividido por vendas).

\subsection{Identificação das empresas comparáveis (peers)}

Peer é a denominação dada para uma empresa ou ativo que é (suficientemente) comparável à empresa ou ao ativo que se quer analisar. A identificação das peers é um dos pontos cruciais em avaliação por múltiplos. Diversos autores têm se dedicado a estudar e testar lógicas para esta identificação.

Existem duas grandes correntes, não necessariamente excludentes, para a identificação de peers, sendo o agrupamento por:

(i) Setor de atuação e

(ii) Fundamentos econômicos semelhantes.

A opção pelo agrupamento por (i) setor de atuação considera que empresas do mesmo setor têm drivers de valor similares. A opção por agrupamento por (ii) fundamento econômico considera que empresas com mesmos drivers de valor não necessariamente são do mesmo setor. $\mathrm{O}$ agrupamento setorial pode ser afunilado, para considerar um subsetor ou para considerar, dentro do setor, aquelas empresas que apresentem determinada característica similar (por exemplo, agrupamento por tamanho dentro do agrupamento setorial). Muitos autores não recomendam formar grupos com menos do que 5 empresas (BOATSMAN; BASKIN, 1981; LIE; LIE, 2002), o que limita tal afunilamento setorial no Brasil, tendo em vista que muitos setores têm baixo número de empresas componentes. 
Os estudos de Alford (1992), Kim e Ritter (1999), Liu, Nissim e Thomas (2002), Lie e Lie (2002), Henschke e Homburg (2009) e Couto Jr. e Galdi (2012) indicam a preferência pelo agrupamento setorial (puro ou com um filtro adicional). Por outro lado, os estudos de Boatsman e Baskin, 1981, Bhojaraj e Lee, 2002, Bhojaraj, Lee e Ng, 2003, Herrmann e Richter, 2003, Dittmann e Maug (2008), Nel, Bruwer e Roux (2014), Young e Zeng (2015) e Serra e Fávero (2017) indicam a preferência por agrupamento por fundamento econômico.

Considerando um fluxo de caixa perpétuo desde o início, pode-se calcular seu preço como proposto na Equação 1 (COPELAND; KOLLER; MURRIN, 2002).

$$
\text { Preço }=\text { Enterprise Value }=\frac{\mathrm{FC}}{(\mathrm{i}-\mathrm{g})}=\frac{\mathrm{ROL} \times(1-\mathrm{g} / \mathrm{r})}{(\mathrm{i}-\mathrm{g})}
$$

Em que FC é o fluxo de caixa da empresa, ROL é o resultado operacional líquido, g é a taxa de crescimento na perpetuidade, $r$ é a rentabilidade (ROIC) e i é o custo de capital (WACC).

O índice EV/EBITDA pode ser calculado como:

$$
\frac{\mathrm{EV}}{\text { EBITDA }}=\frac{\frac{\operatorname{ROL} \times\left(1-\frac{\mathrm{g}}{\mathrm{r}}\right)}{(\mathrm{i}-\mathrm{g})}}{\text { EBITDA }}=\frac{\mathrm{ROL}}{\text { EBITDA }} \times \frac{1}{\mathrm{r}} \times \frac{\mathrm{r}-\mathrm{g}}{(\mathrm{i}-\mathrm{g})}=\frac{\mathrm{ROL}}{\mathrm{EBITDA}} \times \frac{1}{\mathrm{r}} \times\left(1+\frac{\mathrm{r}-\mathrm{i}}{(\mathrm{i}-\mathrm{g})}\right)
$$

Em que EV é o enterprise value e EBITDA é lucro antes dos juros, impostos, depreciação e amortização, sendo os demais itens já definidos. Serra e Fávero (2017) apontam que o termo entre parênteses da Equação 2 é comum aos diversos múltiplos $(\mathrm{P} / \mathrm{E}, \mathrm{P} / \mathrm{B}$, EV/Vendas etc.). Portanto, considerou-se como drivers de valor: rentabilidade (mais rigorosamente, rentabilidade acima do custo de capital ou r-i), custo de capital (i) e crescimento (g). Diversos estudos corroboram a relação destes drivers com valor (WILCOX, 1984; FAIRFIELD, 1994; BHOJRAJ; LEE, 2002; NEL; BRUWER; ROUX; 2014; SERRA; SAITO; 2015).

Além destas características, também se encontra referência para a utilização do filtro por tamanho (ALFORD, 1992; CHENG; MCNAMARA, 2000; BHOJRAJ; LEE, 2002; NEL; BRUWER; ROUX, 2014; SERRA; FÁVERO, 2017), entre outras razões, por poder ser uma proxy de risco (com ressalvas). $\mathrm{O}$ tamanho também se justifica por ser considerado uma variável importante na precificação de IPO’s (LOWRY; SCHWERT, 2004).

\subsection{Processo de precificação no IPO}

A precificação é realizada a partir da leitura da demanda dos investidores pelo banco de investimento que, usualmente, aplica um dos dois processos: bookbuilding ou leilão, sendo o bookbuilding o processo mais usual (SHERMAN, 2005). Cornelli e Goldreich (2001) explicam que no bookbuilding, o banco de investimento solicita aos investidores suas intenções de quantidade e preço máximo de compra e com isso identifica a demanda do mercado. Entretanto é o banco de investimento que define o preço final e realiza a alocação das quantidades para cada um dos investidores que apresentaram suas demandas. De acordo com os autores, esse processo pode não levar ao verdadeiro preço de equilíbrio em função do processo não transparente de alocação das ações aos investidores.

A alternativa ao bookbuilding mais comum é o leilão em que as quantidades e os preços são ofertados e à medida que as demandas vão sendo atendidas, o preço vai caindo até que todas as ações sejam vendidas, eliminando assim a discricionariedade do banco de investimento. Almeida e Leal (2010) realizaram experimento em que avaliam outro processo de precificação, 
o IPO competitivo, caracterizado pela semelhança com o bookbuilding, entretanto, conta com a participação de outros bancos, o que minimiza problemas de discricionariedade do banco responsável pela alocação das quantidades de ações aos investidores. No Brasil, o processo de precificação é realizado, majoritariamente, pelo bookbuilding, o que, segundo Almeida e Leal (2010), leva ao melhor resultado para o investidor e pior resultado para a empresa emissora.

A subprecificação foi identificada, no mercado americano, por Ibbotson (1975) e Ritter (1984) e posteriormente corroborada como um fenômeno não restrito ao mercado de capitais americanos por Loughran, Ritter e Rydqvist (1994). O erro de precificação é justificado pela presença de assimetria de informação entre o emissor e os investidores menos informados, exigindo assim um prêmio pela falta de informação. Beatty e Ritter (1986) e Rock (1986) indicam que a subprecificação é positivamente correlacionada com o nível de incerteza resultante da assimetria de informações entre as partes envolvidas no processo. Outra motivação para a subprecificação é a motivação financeira (BENVENISTE; SPINDT, 1989; BENVENISTE; WILHELM, 1990). Os bancos de investimentos podem utilizar-se deste expediente para motivar investidores a se interessarem pelas ações para garantir que um número suficiente de investidores participe da oferta. Lowry, Officer e Schwert (2010) afirmam que o problema da subprecificação justifica-se pela incerteza sobre a demanda pelos títulos a serem ofertados no mercado, o que pressiona o banco de investimento decidir, conservadoramente, por um preço menor do que o preço justo. Os autores afirmam ainda que o processo de bookbuilding contribui para a assimetria de informação e que o leilão seria o processo ideal para minimizar o problema de subprecificação.

No mercado brasileiro, o fenômeno da incerteza no processo de precificação foi documentado nos IPO's de 2004 a 2007 por Silva e Famá (2011), tal qual Lowry, Officer e Schwert (2010) haviam feito. O fenômeno de subprecificação foi documentado por Leal (1991), Casotti e Motta (2008), que estudaram os IPO's de 2004 a 2006, por meio dos múltiplos P/E e EV/EBIT e por Silva e Famá (2011).

\section{Método}

\subsection{Amostra}

O período de análise foi de 2004 a 2014, totalizando 11 anos. Neste período, ocorreram IPO's de 116 empresas. Foram excluídos da amostra os IPO's de: (i) empresas financeiras (bancos, seguradoras etc.), (ii) empresas com informações negativas (EBITDA, patrimônio líquido ou ativo) ou sem informações completas no prospecto, (iii) empresas sem peers ou empresas com peers com informações faltantes ou negativas (EBITDA, patrimônio líquido ou ativo) e (iv) empresas e peers consideradas outliers (com informação acima ou abaixo de 1,96 desvio-padrão, que para uma distribuição normal representaria $5 \%$ das observações). A amostra totalizou 36 IPO's.

As informações de EBITDA, patrimônio líquido, endividamento e ativos foram obtidas: (i) no prospecto do IPO para as empresas abrindo o capital e (ii) no sistema de informações Economatica $^{\circledR}$ para as empresas comparáveis (peers). O enterprise value foi obtido na Economatica, tanto para os IPO's quanto para as peers.

Foram utilizados os preços das ações em dois momentos: (i) do dia do IPO (preço resultado do processo de bookbuilding) (D0) e (ii) do fechamento do primeiro dia de negociação (D1).

Genericamente, um múltiplo é a razão entre valor (equity value ou enterprise value) e parâmetro (lucro líquido, patrimônio líquido ou EBITDA). Diferentemente do valor - que está disponível diariamente, o parâmetro, por se tratar de uma informação das demonstrações financeiras, não está disponível diariamente. Portanto, o múltiplo é calculado com a informação 
do valor na data da análise e a informação do parâmetro defasada. A data do parâmetro corresponde à data da última demonstração financeira disponível no prospecto (o EBITDA utilizado foi o dos últimos doze meses findos na referida data).

A classificação setorial utilizada foi a da NAICS (North American Industry Classification System). Adicionalmente, analisou-se os dados agrupados pela classificação setorial da Economatica.

\subsection{Variáveis}

Além do múltiplo das peers, outras variáveis também foram utilizadas para explicar o múltiplo da empresa abrindo o capital. As variáveis utilizadas são as consideradas, por alguns autores (WILCOX, 1984; BHOJRAJ; LEE, 2002; SERRA; SAITO, 2015), como sendo as variáveis determinantes dos múltiplos ou de valor (seção 2.3). Neste trabalho utilizou-se: rentabilidade (medido por EBITDA/ativo), alavancagem (como proxy do risco, uma vez que a empresa abrindo o capital não possui beta, medido por dívida/patrimônio líquido) e tamanho (medido pelo logaritmo natural do ativo).

Devido à dificuldade de obter o crescimento histórico das vendas (exigiria que os prospectos tivessem apresentado, por no mínimo 2 anos, as demonstrações financeiras de determinados trimestres e não apenas as anuais), esta variável não foi utilizada. A Tabela 1 sumariza as variáveis consideradas e seus respectivos cálculos.

Tabela 1 - Descrição das variáveis.

\begin{tabular}{lll}
\hline \hline Variável & Nome & Cálculo \\
\hline M_Peer & Múltiplo das peers & $\begin{array}{l}\text { Múltiplo das empresas comparáveis (calculado por média, } \\
\text { mediana e média harmônica) }\end{array}$ \\
rent & Rentabilidade & EBITDA dos últimos 12 meses dividido pelo ativo \\
alav & Alavancagem & $\begin{array}{l}\text { Dívida líquida (endividamento menos caixa) dividido pelo } \\
\text { patrimônio líquido }\end{array}$ \\
tam & Tamanho & Logaritmo natural do ativo \\
\hline Fonte: Elaborada pelos autores.
\end{tabular}

\subsection{Método}

O objetivo do trabalho é verificar se, no momento do IPO, os múltiplos das empresas pertencentes ao mesmo setor da empresa participante do IPO são bons parâmetros para precificá-la. Sendo assim, a hipótese testada é: IPO's.

$H_{0}$ : Os múltiplos das empresas comparáveis não são relevantes na precificação dos

$H_{1}$ : Os múltiplos das empresas comparáveis são relevantes na precificação dos IPO's.

O agrupamento utilizado foi o setorial, por ser o mais usual entre analistas (DAMODARAN, 2006). No Brasil, devido à baixa quantidade de empresas por setor, não se procedeu com um afunilamento dentro do setor. Porém, utilizou-se no modelo variáveis para controlar pela diferença nos drivers de valor (rentabilidade, alavancagem como proxy do risco e tamanho conforme apresentando na Tabela 1 e na Seção 2.3). Para verificar a existência de diferença no comportamento por ano, utilizou-se uma variável dummy de tempo (tendo 2004 como ano base).

O modelo utilizado está expresso na Equação (3).

$$
\begin{aligned}
\mathrm{M}_{-} \mathrm{IPO}_{\mathrm{i}}=\beta_{0}+ & \left(\beta_{1}+\beta_{2} \times \text { dummy_tempo }_{\mathrm{i}}\right) \times \mathrm{M}_{-} \mathrm{Peer}_{\mathrm{i}}+\beta_{3} \times\left(\text { rent }_{-} \mathrm{IPO}_{\mathrm{i}}\right. \\
& \left.- \text { rent_Peer }_{\mathrm{i}}\right)+\beta_{4} \times\left(\text { alav_IPO }_{\mathrm{i}}-\text { alav_Peer }_{\mathrm{i}}\right)+\beta_{5} \times\left(\operatorname{tam}_{-} \mathrm{IPO}_{\mathrm{i}}\right. \\
& \left.- \text { tam_Peer }_{\mathrm{i}}\right)+\varepsilon_{\mathrm{i}}
\end{aligned}
$$


Em que, M_IPO é o múltiplo da empresa i abrindo o capital, M_Peer é o múltiplo das empresas listadas do setor da empresa i abrindo o capital (calculado por três alternativas: média,

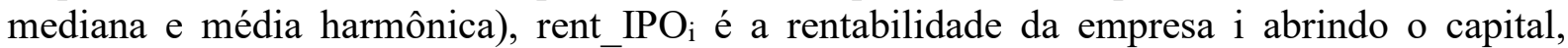
rent_Peer ${ }_{i}$ é a rentabilidade das empresas listadas do setor da empresa i abrindo o capital (calculado por três alternativas: média, mediana e média harmônica), alav_IPO i é a alavancagem da empresa i abrindo o capital, alav_Peeri é a alavancagem das empresas listadas do setor da empresa i abrindo o capital (calculado por três alternativas: média, mediana e média

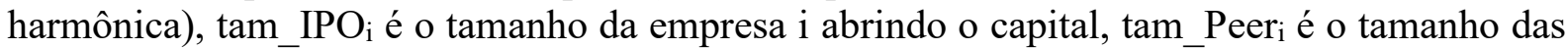
empresas listadas do setor da empresa i abrindo o capital (calculado por três alternativas: média, mediana e média harmônica), dummy_tempo é a variável dummy indicando o ano da abertura de capital, e $\varepsilon_{\mathrm{i}}$ é o termo de erro.

Ao utilizar a diferença das variáveis rentabilidade, alavancagem e tamanho entre as empresas abrindo o capital e suas peers quer-se verificar se a diferença entre seus múltiplos pode ser explicada pelas diferenças em seus drivers de valor.

\section{Análise dos Dados}

\subsection{Estatísticas Descritivas}

A Tabela 2 apresenta a quantidade de IPO's por ano e por setor, já excluídos os anos em que nenhum IPO foi analisado (não compôs a amostra). Verifica-se grande concentração de IPO's em 2007, ano com grande liquidez no mercado de capitais internacional. O setor que apresentou maior número de IPO's foi o setor de construção de edifícios residenciais (incorporadoras), representando uma parcela considerável de todos os IPO's e, em particular, em 2007.

Tabela 2 - Quantidade de IPO por ano e por setor.

\begin{tabular}{lccccccccc} 
& $\mathbf{2 0 0 4}$ & $\mathbf{2 0 0 5}$ & $\mathbf{2 0 0 6}$ & $\mathbf{2 0 0 7}$ & $\mathbf{2 0 0 9}$ & $\mathbf{2 0 1 0}$ & $\mathbf{2 0 1 1}$ & $\mathbf{2 0 1 3}$ & Total \\
\cline { 2 - 9 } Agricultura & 0 & 0 & 0 & 0 & 0 & 0 & 0 & 1 & 1 \\
Abatedouros & 0 & 0 & 0 & 3 & 0 & 0 & 0 & 0 & 3 \\
Indústria de açúcar e produtos de & 0 & 0 & 0 & 2 & 0 & 0 & 0 & 0 & 2 \\
confeitaria & 0 & 0 & 0 & 0 & 0 & 0 & 1 & 0 & 1 \\
Loja de departamentos & 0 & 0 & 3 & 8 & 1 & 0 & 0 & 0 & 12 \\
Construção de edifícios residenciais & 1 & 1 & 0 & 0 & 0 & 0 & 0 & 0 & 2 \\
Geração, transmissão e distribuição de & & & & & & & \\
energia elétrica & 0 & 0 & 0 & 0 & 0 & 0 & 1 & 0 & 1 \\
Extração de petróleo e gás & 0 & 0 & 0 & 1 & 0 & 0 & 0 & 0 & 1 \\
Indústria de fertilizantes e pesticidas & 0 & 0 & 0 & 1 & 0 & 0 & 0 & 0 & 1 \\
Indústria química básica & 0 & 0 & 1 & 0 & 0 & 0 & 0 & 0 & 1 \\
Forjarias e estamparias & 0 & 0 & 0 & 1 & 0 & 0 & 0 & 0 & 1 \\
Telecomunicações & 0 & 0 & 0 & 0 & 0 & 0 & 1 & 0 & 1 \\
Indústria de calçados & 0 & 1 & 0 & 1 & 0 & 1 & 0 & 0 & 3 \\
Atividades auxiliares ao transporte & 1 & 0 & 0 & 0 & 0 & 0 & 0 & 0 & 1 \\
rodoviário & 0 & 0 & 0 & 2 & 0 & 1 & 1 & 0 & 4 \\
Transporte aéreo regular & 0 & 0 & 0 & 0 & 1 & 0 & 0 & 0 & 1 \\
Locadora de imóveis & $\mathbf{2}$ & $\mathbf{2}$ & $\mathbf{4}$ & $\mathbf{1 9}$ & $\mathbf{2}$ & $\mathbf{2}$ & $\mathbf{4}$ & $\mathbf{1}$ & $\mathbf{3 6}$ \\
Laboratório de exames médicos & & & & & & & &
\end{tabular}

A Tabela 3 apresenta a média, mediana, desvio padrão e correlação entre as variáveis analisadas. 
Tabela 3 - Média, mediana, desvio padrão e correlação das variáveis. Significância da correlação entre parênteses.

\begin{tabular}{|c|c|c|c|c|c|c|c|c|c|}
\hline & Média & Mediana & $\begin{array}{l}\text { Desvio } \\
\text { Padrão }\end{array}$ & $\begin{array}{c}\text { M_IPO } \\
\text { (D0) }\end{array}$ & $\begin{array}{c}\text { M_IPO } \\
\text { (D1) }\end{array}$ & $\begin{array}{c}\text { M_Peer } \\
\text { (D0) }\end{array}$ & $\begin{array}{c}\text { M_Peer } \\
\text { (D1) }\end{array}$ & $\begin{array}{l}\text { rent_IPO- } \\
\text { rent_Peer }\end{array}$ & $\begin{array}{l}\text { alav_IPO- } \\
\text { alav_Peer }\end{array}$ \\
\hline M_IPO (D0) & 21,44 & 17,46 & 11,81 & & & & & & \\
\hline M_IPO (D1) & 20,16 & 16,33 & 10,94 & $\begin{array}{c}0,938 \\
(0,000)\end{array}$ & & & & & \\
\hline M_Peer (D0) & 18,44 & 17,88 & 9,89 & $\begin{array}{c}0,643 \\
(0,000)\end{array}$ & $\begin{array}{c}0,597 \\
(0,000)\end{array}$ & & & & \\
\hline M_Peer (D1) & 18,37 & 17,62 & 9,92 & $\begin{array}{c}0,622 \\
(0,000)\end{array}$ & $\begin{array}{c}0,572 \\
(0,000)\end{array}$ & $\begin{array}{c}0,998 \\
(0,000)\end{array}$ & & & \\
\hline $\begin{array}{l}\text { rent_IPO- } \\
\text { rent_Peer }\end{array}$ & 0,06 & 0,03 & 0,16 & $\begin{array}{l}-0,230 \\
(0,177)\end{array}$ & $\begin{array}{l}-0,246 \\
(0,148)\end{array}$ & $\begin{array}{c}0,013 \\
(0,9394)\end{array}$ & $\begin{array}{c}0,019 \\
(0,914)\end{array}$ & & \\
\hline $\begin{array}{l}\text { alav_IPO- } \\
\text { alav_Peer }\end{array}$ & 3,20 & $-0,05$ & 17,70 & $\begin{array}{l}-0,093 \\
(0,589)\end{array}$ & $\begin{array}{l}-0,073 \\
(0,672)\end{array}$ & $\begin{array}{l}-0,041 \\
(0,813)\end{array}$ & $\begin{array}{l}-0,041 \\
(0,811)\end{array}$ & $\begin{array}{l}-0,233 \\
(0,172)\end{array}$ & \\
\hline $\begin{array}{l}\text { tam_IPO- } \\
\text { tam_Peer }\end{array}$ & $-0,79$ & $-0,92$ & 1,25 & $\begin{array}{c}0,006 \\
(0,974)\end{array}$ & $\begin{array}{c}0,125 \\
(0,469)\end{array}$ & $\begin{array}{c}0,145 \\
(0,399) \\
\end{array}$ & $\begin{array}{c}0,144 \\
(0,402)\end{array}$ & $\begin{array}{l}-0,119 \\
(0,491) \\
\end{array}$ & $\begin{array}{c}0,024 \\
(0,889)\end{array}$ \\
\hline
\end{tabular}

As distribuições dos múltiplos dos IPO's em D0 (dia do IPO) e em D1 (primeiro dia de negociação), dos múltiplos médios dos peers em D0 e em D1 e da diferença do tamanho (tam_IPO - tam_Peer) são normais (valores p do teste de Kolmogorov-Smirnov (K-S) são, respectivamente, $0,360,0,303,0,753,0,736$ e 0,120). As distribuições da diferença das rentabilidades (rent_IPO - rent_Peer) e da diferença do tamanho (alav_IPO - alav_Peer) não são normais (valores p do teste K-S são, respectivamente, 0,014 e 0,000 ).

Embora verifique-se, na Tabela 3, que o múltiplo médio (EV/EBITDA) dos IPO's caiu aproximadamente 1,28x (de 21,44x para 20,16x) entre o dia D0 (dia do IPO) e o dia D1 (primeiro dia de negociação), esta diferença não é estatisticamente significativa a 5,0\% (valor $\mathrm{p}$ do teste $\mathrm{t}$ é 0,071$)$. O mesmo pode-se dizer da diferença entre os múltiplos médios dos peers do dia do IPO e o primeiro dia de negociação (valor $\mathrm{p}$ do teste t é 0,508 ). Pode-se verificar, também, que múltiplo médio dos IPO's é maior do que o múltiplo médio dos peers, tanto em D0 $(21,44 x$ versus $18,44 x)$ como em D1 $(20,16 x$ versus $18,37 x)$, porém, sem significância estatística (valores $p$ do teste $t$ são, respectivamente, 0,062 e 0,274). A manutenção do nível dos múltiplos está em linha com Casotti e Motta (2008), que não observaram subavaliação dos IPO's e em contraposição a Silva e Famá (2011), que, por meio de estudo de eventos, identificaram subavaliação em períodos de tempo parecidos (2004-2006 e 2004-2007, respectivamente).

As diferenças de rentabilidade média (rent_IPO - rent_Peer) e tamanho médio (tam_IPO - tam_Peer) entre as empresas abrindo o capital e as peers, por sua vez, são estatisticamente significativas (valor $\mathrm{p}$ do teste Wilcoxon é 0,01 para a diferença de rentabilidade e o valor $\mathrm{p}$ do teste $\mathrm{t}$ é 0,00 para a diferença de tamanho). A diferença de alavancagem média (alav_IPO alav_Peer) é estatisticamente zero (valor $p$ do teste Wilcoxon é 0,85). No entanto, economicamente, pode-se dizer que existem diferenças nestes três drivers de valor entre as empresas abrindo o capital e as peers. Estas 3 diferenças colaboram para o equity story da abertura de capital pois, em média, as empresas abrindo o capital apresentam maior rentabilidade que suas peers e só não 'fazem mais' (crescem ou são maiores) por falta de capital (estão mais alavancadas) - o que favorece a captação de novos recursos (por meio de IPO primário, ou seja, com entrada de novos recursos na empresa).

No que diz respeito às correlações, verifica-se que os múltiplos das empresas abrindo o capital têm correlação com os múltiplos de suas peers (tanto para D0 quanto para D1), porém, não têm correlação com a diferença dos drivers de valor ou proxy deles (rentabilidade, alavancagem e tamanho). Entre as diferenças dos drivers, não se verifica correlações significativas. 


\subsection{Regressão Linear}

Foram realizadas análises, por meio da Equação 3, para verificar se os múltiplos das empresas pertencentes ao mesmo setor da empresa participante do IPO são bons parâmetros para precificá-la no seu IPO. Foram estimados modelos a partir da equação total (com todas as variáveis explicativas) ou da equação parcial (sem todas as variáveis explicativas), em um total de 3 modelos: (I) considerando apenas os múltiplos das peers como variável explicativa, (II) considerando, além dos múltiplos das peers, as variáveis dummy de ano e (III) o modelo completo.

Cada um dos três modelos foi estimado para (a) o dia D0 e (b) o dia D1. Além disto, as variáveis das peers (múltiplo, rentabilidade, alavancagem e tamanho) foram calculadas por meio de três diferentes métodos: (i) a média, (ii) a mediana e (iii) a média harmônica. Como nenhuma dummy de ano nem variável de diferença de driver (rentabilidade, alavancagem e tamanho) foi significativa, a Tabela 4 apresenta apenas o Modelo I. O único Modelo II apresentado justifica-se pela única exceção em que as variáveis dummy de ano mostraram-se significativas: modelo de D1 para média harmônica.

Tabela 4 - Resultado dos modelos estimados a partir da Equação 3. Significância dos coeficientes entre parênteses.

\section{Painel (a): D0 (dia do IPO)}

\begin{tabular}{lccc} 
& (i) Média & (ii) Mediana & $\begin{array}{c}\text { (iii) Média } \\
\text { Harmônica }\end{array}$ \\
\cline { 2 - 4 } & Modelo I & Modelo I & Modelo I \\
\cline { 2 - 4 } M_Peer & 0,7685 & 0,7271 & 0,7491 \\
Intercepto & $(0,000)$ & $(0,000)$ & $(0,000)$ \\
$\mathrm{R}^{2}$ & 7,8926 & 8,3483 & 9,1178 \\
Reset Test & $(0,033)$ & $(0,010)$ & $(0,008)$ \\
\hline
\end{tabular}

Painel (b): D1 (primeiro dia de negociacão)

\begin{tabular}{lcccc} 
& (i) Média & (ii) Mediana & \multicolumn{2}{c}{ (iii) Média Harmônica } \\
\cline { 2 - 5 } & Modelo I & Modelo I & Modelo I & Modelo II \\
\cline { 2 - 5 } M_Peer & 0,6311 & 0,6103 & 0,5907 & \\
Dummy 2006 x M_Peer & $(0,000)$ & $(0,000)$ & $(0,001)$ & 0,5565 \\
& & & & $(0,005)$ \\
Dummy 2007 x M_Peer & & & 0,4412 \\
& & & & $(0,004)$ \\
Intercepto & 8,5687 & 9,1944 & 10,4273 & 14,0501 \\
R ${ }^{2}$ Ajustado & $(0,012)$ & $(0,004)$ & $(0,002)$ & $(0,000)$ \\
VIF & 0,3276 & 0,3412 & 0,2709 & 0,2647 \\
Reset Test & & & & 1,08 \\
\hline
\end{tabular}

Observa-se, na Tabela 4, que em todos os modelos, os múltiplos das peers mostraramse significativos (com valores p abaixo de $0,1 \%$ ). No caso do modelo em D1 a partir da média harmônica, a significância do múltiplo das peers deu-se apenas nos 2 anos em que a iteração da dummy de tempo mostrou-se significativa (2006 e 2007, anos com maior número de IPO's). Os modelos de média harmônica são os que apresentaram menor fator de ajuste (medido pelo $\mathrm{R}^{2}$ ), em ambos os dias (D0 e D1). Para D0 (painel (a)), os modelos a partir da média e da mediana 
apresentam os maiores fatores de ajuste $\left(\mathrm{R}^{2}\right)$. Para D1 (painel (b)), o melhor fator de ajuste deuse para o modelo a partir da mediana.

O teste RESET (hipótese nula de não omissão de variáveis) indicou que todos os modelos não apresentam variáveis omitidas (a 5\% de significância). Para o modelo em D1 a partir da média harmônica, que apresentou mais de uma variável significativa, o baixo variance inflation factor (VIF) indica que as variáveis explicativas não apresentam problemas de multicolinearidade. Para este modelo, o teste RESET foi apresentado apenas para o modelo mais completo. No entanto, nota-se que, ignoradas as dummys de tempo, o múltiplo das peers foi significativo na explicação do múltiplo dos IPO's.

Os coeficientes da variável M_Peer são menores do que 1,0 (Tabela 4) indicando que cada unidade de múltiplo das peers é refletido com desconto nos múltiplos dos IPO's, não obstante os múltiplos médios dos IPO's serem estatisticamente iguais aos múltiplos médios das peers (Tabela 3 ).

Considerando (a) as diferenças nos drivers de valor entre os IPO's e as peers identificadas na seção 4.1 e a literatura indicando (b1) que filtros por drivers de valor ajudam na identificação de peers (KIM; RITTER, 1999; LIE; LIE, 2002; COUTO JR.; GALDI, 2012) ou (b2) que o agrupamento por fundamentos econômicos (BOATSMAN; BASKIN, 1981; BHOJARAJ; LEE, 2002; NEL; BRUWER; ROUX, 2014; SERRA; FÁVERO, 2017) é melhor para a identificação de peers, seria esperado que as variáveis ligadas às diferenças de drivers fossem significativas na explicação dos múltiplos, o que não se verificou. Nem mesmo tamanho, identificado como variável importante na precificação de IPO's (LOWRY; SCHWERT, 2004), mostrou-se significativo. A ausência de ajustes por drivers de valor reflete na igualdade estatística dos múltiplos dos IPO's e dos múltiplos das peers.

Os resultados da Tabela 4 permitem confirmar a hipótese de que os múltiplos das empresas comparáveis são relevantes na precificação dos IPO's, conforme proposto pela pesquisa.

\subsection{Classificação Setorial Economatica}

A análise foi repetida com a classificação setorial da Economatica (21 setores). Considerou-se na amostra 31 empresas, tendo-se retirado 5 empresas classificadas no setor Outros da Economatica, que compunham a amostra de 36 empresas utilizada na análise da classificação setorial NAICS. Apenas o modelo com uma única variável explicativa (M_Peer) foi estimado. Os IPO's estão distribuídos em 11 setores pela classificação da Economatica e por 14 setores na classificação NAICS (2 a menos do que indicado na Tabela 2).

Tabela 5 - Resultado dos modelos estimados, considerando apenas a variável explicativa M Peer da Equação 3. Duas classificações setoriais foram consideradas: Economatica (Econ) e NAICS. Significância dos coeficientes entre parênteses.

\section{Painel (a): D0 (dia do IPO)}

\begin{tabular}{lccccccc} 
& \multicolumn{2}{c}{ (i) Média } & \multicolumn{2}{c}{ (ii) Mediana } & \multicolumn{2}{c}{$\begin{array}{c}\text { (iii) Média } \\
\text { Harmônica }\end{array}$} \\
\cline { 2 - 8 } & Econ & NAICS & Econ & NAICS & \multicolumn{2}{c}{ Econ } & NAICS \\
\cline { 2 - 8 } M_Peer & 0,9844 & 0,7744 & 0,5841 & 0,7337 & 1,099 & 0,7514 \\
& $(0,001)$ & $(0,000)$ & $(0,020)$ & $(0,000)$ & $(0,045)$ & $(0,000)$ \\
Intercepto & 5,2839 & 5,9484 & 12,0688 & 7,0479 & 9,0506 & 7,9678 \\
R $^{2}$ Ajustado & $(0,225)$ & $(0,075)$ & $(0,005)$ & $(0,027)$ & $(0,130)$ & $(0,020)$ \\
Reset Test & 0,3445 & 0,4784 & 0,1737 & 0,4798 & 0,1317 & 0,4105 \\
& 0,4337 & 0,0014 & 0,4764 & 0,0296 & 0,6189 & 0,0342 \\
\hline
\end{tabular}


Tabela 5 - Resultado dos modelos estimados, considerando apenas a variável explicativa M_Peer da Equação 3. Duas classificações setoriais foram consideradas: Economatica (Econ) e NAICS. Significância dos coeficientes entre parênteses.

Painel (b): D1 (primeiro dia de negociação)

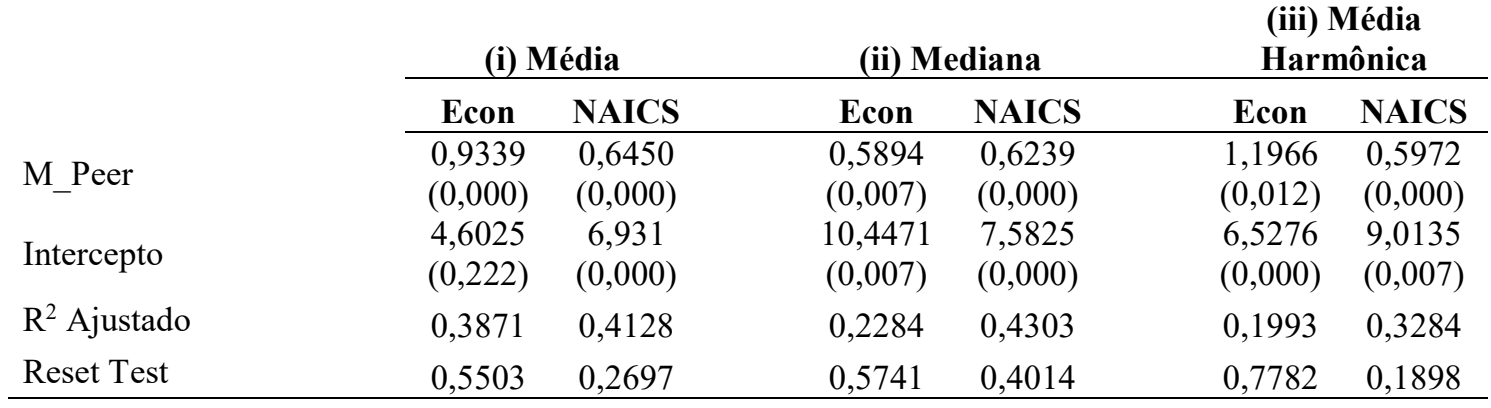

Observa-se na Tabela 5 que os modelos com a classificação NAICS produzem melhores coeficientes de ajustes $\left(\mathrm{R}^{2}\right)$ do que os modelos com a classificação Economatica, sugerindo que, para o propósito em questão, aquela classificação setorial seria melhor do que esta. Os coeficientes dos múltiplos das peers têm, no conjunto, significância ligeiramente melhor nos modelos NAICS.

Por fim, pode-se observar que os modelos NAICS indicam consistência em apontar a relação abaixo de 1,0 dos múltiplos dos IPO's em relação aos múltiplos das peers (coeficientes menores do que 1), além de apresentarem coeficientes mais estáveis (para os modelos de média, mediana e média harmônica), o que não se observa nos modelos a partir da classificação setorial da Economatica. Este resultado está em linha com a escolha de Casotti e Motta (2008) pela classificação NAICS no seu estudo de múltiplos e de Serra (2018) pela classificação SIC no seu estudo de betas, ambos estudos envolvendo a escolha de peers.

\section{Considerações Finais}

A precificação de empresas abrindo o capital é uma tarefa crítica no processo de IPO. Dada a menor disponibilidade de informações históricas das empresas abrindo o capital comparativamente às empresas listadas e estabelecidas, a avaliação por múltiplos pode ser favorecida nestas ocasiões, sendo bastante utilizada e recomendada (KIM; RITTER, 1999; CASOTTI; MOTTA, 2008), sem negligenciar a aplicação da técnica avaliação por fluxo de caixa descontado.

Analisando o múltiplo EV/EBITDA de 36 IPO's na Bolsa de Valores de São Paulo no período de 2004 a 2014 (onze anos) verificou-se que os múltiplos das empresas comparáveis (peers, considerando-se como peer empresa do mesmo setor conforme classificação da NAICS - North American Industry Classification System) são relevantes na precificação dos IPO's, em linha com resultados apresentados por Kim e Ritter (1999) e Firth et al. (2008). Tal resultado colabora com as evidências empíricas da relevância da avaliação por múltiplos no processo de IPO e, eventual e mais genericamente, para empresas de capital fechado. Os múltiplos dos IPO's foram calculados exclusivamente com informações existentes na época de cada IPO, que seriam as informações que os investidores teriam disponíveis para fazerem suas análises, ou seja, não foram consideradas informações 'futuras' conhecidas apenas após o IPO (dados expost).

Os modelos com múltiplos médios e medianos apresentaram melhor ajuste (medido pelo $\mathrm{R}^{2}$ ) do que os modelos com múltiplos a partir da média harmônica. Em adição, no fechamento do primeiro dia de negociação, o modelo da média harmônica (alternativa à média) não indicou significância para o múltiplo das empresas comparáveis para todos os anos da amostra (apenas para 2006 e 2007, por meio da variável dummy de ano). 
Foram analisados dois momentos distintos: (i) o dia do IPO (D0, base para o preço inicial do IPO, o qual é referenciado no prospecto final do IPO) e (ii) o fechamento do primeiro dia de negociação (D1). Os modelos em D0 apresentaram melhores coeficientes de ajuste (medido pelo $\mathrm{R}^{2}$ ), o que pode indicar maior alinhamento na precificação das empresas abrindo o capital com as peers no bookbuilding comparativamente ao final do primeiro dia de negociação.

Também foi possível verificar que as empresas que abriram o capital nos onze anos analisados (2004 a 2014) tinham (a) maior rentabilidade (estatisticamente significativa e medida como EBITDA dividido pelo ativo) histórica do que as peers, (b) maior alavancagem (apenas do ponto de vista econômico e medida como dívida líquida dividido pelo patrimônio líquido) do que as peers e (c) menor base de ativos (estatisticamente significativa) do que a base média das peers.

Esta combinação pode indicar que as empresas que abrem o capital são aquelas com equity story favorecido para captar recursos primários (novos recursos na empresa): a empresa abrindo o capital é melhor operacionalmente do que suas peers listadas (melhor rentabilidade operacional), porém são menores e, portanto, com capacidade de crescimento e só não o fazem por falta de capital (estando inclusive, acima da alavancagem média do setor) - sendo assim, uma injeção de capital próprio impulsionaria seu crescimento. No entanto, as diferenças de tamanho e alavancagem não apoiam o fato de os múltiplos das empresas abrindo o capital serem maiores do que os múltiplos de suas peers (sem significância estatística, Tabela 2).

O maior risco das empresas abrindo o capital (mais alavancadas e menores) indicaria o contrário. Pode ser que os maiores múltiplos das empresas abrindo o capital traduzam a percepção que o mercado tem de que tais empresas têm maior potencial de crescimento do que as empresas listadas e estabelecidas e/ou seja decorrência do processo de precificação do IPO. Por fim, a diferença destes drivers de valor (entre a empresa abrindo o capital e suas peers) não são estatisticamente significantes na explicação da diferença entre os múltiplos das empresas abrindo o capital e suas peers.

Os modelos com a classificação da Economatica apresentaram menores coeficientes de ajuste (medido pelo $\mathrm{R}^{2}$ ) comparados àqueles com a classificação setorial da NAICS (Tabela 5). Este melhor resultado é reflexo do maior número de setores da NAICS, que favorece o agrupamento de empresas mais homogêneas.

Os investidores, portanto, parecem (1) basear-se em informações existentes no prospecto para realizarem suas análises por múltiplos, (2) utilizar múltiplos de peers calculados por meio de média ou mediana, sendo a média harmônica uma medida ainda a ser melhor explorada e (3) serem capazes de identificar as peers melhor do que apenas a classificação da Economatica, tendo em vista que a classificação NAICS (que apresentaram melhores coeficientes de ajustes) seria um filtro maior por considerar uma maior gama de setores. No entanto, parece não se preocupar com ajustes nos múltiplos por diferenças nos drives de valor existentes entre os IPO's e as peers, a não ser que a falta de um driver de crescimento nas análises promovidas no presente artigo esteja obscurecendo tal constatação.

Tais ajustes seriam, em tese, justificáveis, pois diferenças nos drivers indicariam diferenças nos preços e, portanto, nos múltiplos. Por fim, (i) os múltiplos médios dos IPO's e das peers são estatisticamente iguais, embora (ii) o coeficiente menor do que 1,0 da regressão indique que variações de 1 unidade de múltiplo das peers são apenas parcialmente repassadas para os múltiplos IPO's. Estes dois últimos resultados não caminham na mesma direção da confirmação de um desconto na precificação dos IPO's.

Futuras pesquisas podem considerar outros múltiplos e também outras variáveis explicativas (ou outra forma de medir as mesmas variáveis, por exemplo, alavancagem) além da diferença entre os drivers de valor (ou proxy deles) utilizados neste estudo (rentabilidade, alavancagem e tamanho). 


\section{Referências}

ALFORD, A. W. The effect of the set of comparable firms on the accuracy of the priceearnings valuation method. Journal of Accounting Research, v. 30, n. 1, p. 94-108, 1992. DOI: $10.2307 / 2491093$.

ALMEIDA, V. S.; LEAL, R. P. C. Apreçamento em Aberturas de Capital: uma análise experimental do leilão, bookbuilding e IPO competitivo. In $34^{\circ}$ Encontro Nacional da ANPAD, 2010. ANAIS..., 2010.

BAKER, M.; RUBACK, R. Estimating industry multiples. Harvard University. Working paper, 1999.

BALL, R. Anomalies in relationships between securities' yield and yield-surrogates. Journal of Financial Economics, v. 6, n. 2, p. 103-126, 1978. DOI: 10.1016/0304-405X(78)90026-0.

BENVENISTE, L. M.; SPINDT, P. A. How investment bankers determine the offer price and allocation of new issues. Journal of Financial Economics, v. 24, n. 2, p. 343-361, 1989. DOI: $10.1016 / 0304-405 X(89) 90051-2$.

BENVENISTE, L. M.; WILHELM, W. J. A comparative analysis of IPO proceeds under alternative regulatory environments. Journal of Financial Economics, v. 28, n. 1-2, p. 173207, 1990. DOI: 10.1016/0304-405X(90)90052-2.

BHOJRAJ, S.; LEE, C. M. Who is my peer? A valuation-based approach to the selection of comparable firms. Journal of Accounting Research, v. 40, n. 2, p. 407-439, 2002. DOI: 10.1111/1475-679X.00054.

BHOJRAJ, S.; LEE, C. M.; NG, D. T. International valuation using smart multiples. Cornell University. Working paper, 2003.

BLACK, F. Capital Market Equilibrium with Restricted Borrowing. Journal of Business, v. 45, n. 3, p. 444-455, 1972.

BEATTY, R. P.; RITTER, J. R. Investment banking, reputation and the underpricing of initial public offerings. Journal of Financial Economics, v. 15, n. 1-2, p. 213-232, 1986. DOI: $10.1016 / 0304-405 X(86) 90055-3$.

BLACK, F. Beta and return. Journal of Portfolio Management, v. 20, p. 8-18, 1993.

BOATSMAN, J. R.; BASKIN, E. F. Asset valuation with incomplete markets. Accounting Review, v. 1, n. 1, p. 38-53, 1981.

CASOTTI, F. P.; da MOTTA, L. F. J. Oferta pública inicial no Brasil (2004-2006): uma abordagem da avaliação através de múltiplos e do custo de capital próprio. Revista Brasileira de Finanças, v. 6, n. 2, p. 157-204, 2008.

CHAN, L. K.; HAMAO, Y.; LAKONISHOK, J. Fundamentals and stock returns in Japan. Journal of Finance, v. 46, n. 5, p. 1739-1789, 1991. DOI: 10.1111/j.15406261.1991.tb04642.x

CHENG, C. A.; MCNAMARA, R. The valuation accuracy of the price-earnings and pricebook benchmark valuation methods. Review of Quantitative Finance and Accounting, v. 15 , n. 4 , p. 349-370, 2000.

COUTO JR., C. G.; GALDI, F. C. Avaliação de Empresas por Múltiplos Aplicado em Empresas Agrupadas com Análise de Cluster. Revista de Administração Mackenzie, v. 13, n. 5, p. 135-170, 2012. 
CORNELLI, F.; GOLDREICH, D. Bookbuilding and Strategic Allocation. Journal of Finance, v. 56, n. 6, p. 2337-2369, 2001. DOI: 10.1111/0022-1082.00407.

DAMODARAN, A. Valuation approaches and metrics: a survey of the theory and evidence. Now Publishers Inc, 2006. DOI: 10.1561/0500000013.

DITTMANN, I.; MAUG, E. G. Biases and error measures: How to compare valuation methods. ERIM Report Series, Reference No. ERS-2006-011-F\&A, 2006-07, 2008.

FAIRFIELD, P. M. P/E, P/B and the present value of future dividends. Financial Analysts Journal, v. 50, n. 4, p. 23-31, 1994. DOI: 10.2469/faj.v50.n4.23.

FAMA, E. F.; FRENCH, K. R. The cross-section of expected stock returns. Journal of Finance, v. 47, n. 2, p. 427-465, 1992.

FERNANDEZ, P. Valuation using multiples: How do analysts reach their conclusions? (October 18, 2017). SSRN, 2015. http://dx.doi.org/10.2139/ssrn.274972

FIRTH, M.; LI, Y.; WANG, S. S. Valuing IPOs using price-earnings multiples disclosed by IPO firms in an emerging capital market. Review of Pacific Basin Financial Markets and Policies, v. 11, n. 3, p. 429-463, 2008. DOI: 10.1142/S0219091508001428.

HENSCHKE, S.; HOMBURG, C. Equity valuation using multiples: controlling for differences between firms. SSRN, 2009. DOI: 10.2139/ssrn.1270812.

HERRMANN, V.; RICHTER, F. Pricing with performance-controlled multiples. Schmalenbach Business Review, v. 55, n. 3, p. 194-219, 2003. DOI: 10.1007/BF03396674.

IBBOTSON, R. G. Price Performance of Common Stock New Issues. Journal of Financial Economics, v. 2, n. 3, p. 235-272, 1975. DOI: 10.1016/0304-405X(75)90015-X.

JEGADEESH, N.; TITMAN, S. Profitability of momentum strategies: an evaluation of alternative explanations. Journal of Finance, v. 56, n. 2, p. 699-720, 2001. DOI: 10.1111/0022-1082.00342.

KAPLAN, S. N.; RUBACK, R. The valuation of cash flow forecasts: an empirical analysis. Journal of Finance, v. 50, n. 4, p. 1059-1093, 1995. DOI: 10.1111/j.15406261.1995.tb04050.x.

KIM, M.; RITTER, J. R. Valuing IPOs. Journal of Financial Economics, v. 53, n. 3, p. 409437, 1999. DOI: 10.1016/S0304-405X(99)00027-6.

LIE, E.; LIE, H. J. Multiples used to estimate corporate value. Financial Analysts Journal, v. 58, n. 2, p. 44-54, 2002. DOI: 10.2469/faj.v58.n2.2522.

LINTNER, J. The Valuation of Risk Assets and the Selection of Risky Investments in Stock Portfolios and Capital Budgets. Review of Economics and Statistics, v. 47, n. 1, p. 13-37, 1965. DOI: 10.1016/B978-0-12-780850-5.50018-6.

LIU, J.; NISSIM, D.; THOMAS, J. Equity valuation using multiples. Journal of Accounting Research, v. 40, n., 1, p. 135-172, 2002. DOI: 10.1111/1475-679X.00042.

LOUGHRAN, T.; WELLMAN, J. W. New evidence on the relation between the enterprise multiple and average stock returns. Journal of Financial and Quantitative Analysis, v. 46, n. 6, p. 1629-1650, 2012. DOI: 10.1017/S0022109011000445.

LOUGHRAN, T.; RITTER, J. R.; RYDQVIST, K. Initial Public Offerings: International Insights. Pacific-Basin Finance Journal, v. 2, n. 2-3, p. 165-199, 1994. DOI: 
10.1016/0927-538X(94)90016-7.

LOWRY M.; OFFICER, M. S.; SCHWERT, G. W. The Variability of IPO Initial Returns. Journal of Finance, v. 65, n. 2, p. 425-465, 2010. DOI: 10.1111/j.1540-6261.2009.01540.x.

LOWRY M.; SCHWERT, G. W. Is the IPO process efficient? Journal of Financial Economics, v. 71, n. 1, p. 3-26, 2004. DOI: 10.1016/S0304-405X(03)00205-8.

MALKIEL, B. G. Is smart beta really smart? Journal of Portfolio Management, v. 40, n. 5, p. 127-134, 2014.

MCLEAN, R. D.; PONTIFF, J. Does academic research destroy stock return predictability? Journal of Finance, v. 71, n. 1, p. 5-32, 2016. DOI: 10.1111/jofi.12365.

NEL, S.; BRUWER, W.; LE ROUX, N. An emerging market perspective on peer group selection based on valuation fundamentals. Applied Financial Economics, v. 24, n. 9, p. 621637, 2014. DOI: 10.1080/09603107.2014.894629. DOI: 10.1080/09603107.2014.894629.

PENMAN, S. H. The Articulation of Price-Earnings Ratios and Market-to-Book Ratios and the Evaluation of Growth (Digest Summary). Journal of Accounting Research, v. 34, n. 2, p. 235-59, 1996. DOI: $10.2307 / 2491501$.

REINGANUM, M. R. Misspecification of capital asset pricing: Empirical anomalies based on earnings' yield and market value. Journal of Financial Economics, v. 9, n. 1, p. 19-46, 1981. DOI: $10.1016 / 0304-405 X(81) 90019-2$.

RITTER, J. R. The 'hot issue' market of 1980. Journal of Business, v. 57, n. 2, p. 215-240, 1984.

RITTER, J. R. The long-run performance of initial public offerings. Journal of Finance, v. 46, n. 1, p. 3-27, 1991. DOI: 10.1111/j.1540-6261.1991.tb03743.x.

ROCK, K. Why new issues are underpriced? Journal of Financial Economics, v. 15, n. 1-2, p. 187-212, 186. DOI: 0.1016/0304-405X(86)90054-1

SALIBA, R. V. Aplicação de modelos de avaliação por múltiplos no Brasil. Revista Brasileira de Finanças, v. 6, n. 1, p. 13-47, 2008.

SCHWERT, G. W. Anomalies and market efficiency. Handbook of the Economics of Finance, v. 1, p. 939-974, 2003. DOI: 10.1016/S1574-0102(03)01024-0.

SERRA, R. G. An application of hierarchical linear models to analyse Brazilian financial betas. International Journal of Multivariate Data Analysis, v. 1., n. 3., p. 218-229, 2018. DOI: 10.1504/IJMDA.2018.091849.

SERRA, R. G.; WICKERT, M. Valuation: Guia Fundamental. São Paulo: Editora Atlas, 2014.

SERRA, R. G.; FÁVERO, L. P. L. Multiples' valuation: the selection of cross-border comparable firms. Emerging Markets Finance and Trade, 2017. DOI: 10.1080/1540496X.2017.1336084.

SERRA, R. G.; SAITO, A. Revista de Administração Faces Journal, v. 15, n. 1, p. 82-96, 2016. DOI: 10.21714/1984-6975FACES2016V15N1ART2992.

SHARPE, W. F. Capital Asset Prices: A Theory of Market Equilibrium Under Conditions of Risk. Journal of Finance, v. 19, n. 3, p. 425-442, 1964. DOI: 10.1111/j.1540-

6261.1964.tb02865.x. 
SHERMAN, A. E. Global trends in IPO methods: book building versus auctions with endogenous entry. Journal of Financial Economics, v. 78, n. 3, p. 615-649, 2005. DOI: 10.1016/j.jfineco.2004.09.005.

SILVA, J. M. A; FAMÁ, R. Evidencias de Retornos Anormais nos Processos de IPO na Bovespa no período de 2004 até 2007: um estudo de evento. Revista de Administração da USP, v. 46, n. 2, p. 178-190, 2011. DOI: 10.5700/rausp1006.

STATTMAN, D. Book values and stock returns. The Chicago MBA: A Journal of Selected Papers, v. 4, p. 25-45, 1980.

WALKSHÄUSL, C.; LOBE, S. The Enterprise Multiple Investment Strategy: International Evidence. Journal of Financial and Quantitative Analysis, v. 50, n. 4, p. 781-800, 2015. DOI: $10.1017 / \mathrm{S} 002210901500023 \mathrm{X}$.

WILCOX, J. W. The P/B-roe valuation model. Financial Analysts Journal, v. 40, n. 1, p. 58-66, 1984. DOI: 10.2469/faj.v40.n1.58.

YOUNG, S.; ZENG, Y. Accounting comparability and the accuracy of peer-based valuation models. The Accounting Review, v. 90, n. 6, p. 2571-2601, 2015. DOI:10.2308/accr-51053.

ZAROWIN, P. What determines earnings-price rations: revisited. Journal of Accounting, Auditing and Finance, v. 5, n. 3, p. 439-457, 1990. 